\title{
Endocrinology and oncology: closer ties will be mutually beneficial
}

\author{
P Reed Larsen
}

Referral of a patient with thyroid carcinoma to an oncologist for chemotherapy often indicates a serious clinical situation, with palliation sometimes the major goal. Such a step occurs early in the care of patients with anaplastic carcinoma, as these lesions are often extremely aggressive and do not concentrate radioiodine. Referral is less common in patients with well-differentiated carcinoma, however, and usually occurs when the tumor is progressive (despite suppression of TSH levels) and no longer concentrates radioiodine. Chemotherapy with doxorubicin, administered alone or in combination with other agents, is generally used but can be associated with significant toxicity. Clearly then, new therapeutic approaches are needed for patients with progressive thyroid carcinoma.

Reports from phase II trials suggest that the use of anti-angiogenic, small molecule, receptor tyrosine kinase inhibitors (RTKIs), such as axitinib, sorafenib and motesanib, might be useful in this setting. The results of these preliminary trials provide a real basis for optimism. Around $30-50 \%$ of patients with progressive disease (primarily advanced papillary carcinoma) display disease stabilization or, less commonly, decreases in lesion size after treatment with RTKIs. Serum thyroglobulin concentrations often decrease as well, although it is unclear whether this change is caused by a reduction in tumor mass. Such beneficial effects were all too rare with the previous oncologic approaches; however, it is important to acknowledge that RTKIs are also associated with toxic effects. Careful patient selection, improved criteria for pretrial disease progression, and randomized prospective studies are required before the use of RTKIs becomes routine practice. Nonetheless, these encouraging results provide the first evidence that RTKIs might benefit patients with thyroid malignancies.

In addition to the potential benefits of RTKIs, several adverse effects are also relevant for the
RTKls represent both a potential

therapy

for thyroid

carcinoma

refractory to

endocrinologic

approaches

and a newly

recognized

cause of

hypothyroidism... .

$P R$ Larsen is the Editor-in-Chief of Nature Clinical Practice Endocrinology \& Metabolism.

\section{Competing interests}

The author declared no competing interests.

www.nature.com/clinicalpractice doi:10.1038/ncpendmet0974 endocrinologist. Over 50\% of patients receiving sunitinib or sorafenib for gastrointestinal stromal tumors or renal-cell carcinoma develop some degree of primary hypothyroidism. The mechanism for this complication has not been elucidated but several lines of evidence suggest that the anti-angiogenic properties of these drugs might be involved. Such evidence includes the high vascularity of the normal thyroid gland, an association with transient thyroiditis or suppressed TSH levels before onset of hypothyroidism, the complete disappearance of normal thyroid tissue on ultrasound in a few well-documented cases, and the lack of major in vitro effects (at least of sunitinib) on thyroid cells in culture. The possibility of hypothyroidism should be kept in mind, therefore, when evaluating the common symptom of fatigue in patients receiving RTKIs, even though this symptom might occur independently of overt hypothyroidism.

A second, well-documented effect of RTKIs, which has been observed in patients with thyroid carcinoma, is an increase in levothyroxine dose requirements. The mechanism for this effect is also unknown but could reflect impaired absorption of levothyroxine, blockade of $\mathrm{T}_{4}$ activation, or even consumptive hypothyroidism as a result of induction of the thyroid-hormoneinactivating type 3 deiodinase. Although this adverse effect can occur in any levothyroxinetreated patient administered an RTKI, it is especially critical to recognize it in patients with thyroid carcinoma. The resultant elevation of the TSH concentration might attenuate the potential inhibitory effect of the RTKI on the thyroid cancer cells.

In light of these observations, it is vital that endocrinologists become familiar with these novel therapeutic agents. RTKIs represent both a potential therapy for thyroid carcinoma refractory to endocrinologic approaches and a newly recognized cause of hypothyroidism, even in patients already receiving levothyroxine. 